\title{
Exact Description of Rotational Waves in an Elastic Solid
}

\author{
R. A. Close
}

\begin{abstract}
Conventional descriptions of transverse waves in an elastic solid are limited by an assumption of infinitesimally small gradients of rotation. By assuming a linear response to variations in orientation, we derive an exact description of a restricted class of rotational waves in an ideal isotropic elastic solid. The result is a nonlinear equation expressed in terms of Dirac bispinors. This result provides a simple classical interpretation of relativistic quantum mechanical dynamics. We construct a Lagrangian of the form $\mathscr{L}=-\mathscr{E}+U+K=0$, where $\mathscr{E}$ is the total energy, $U$ is the potential energy, and $K$ is the kinetic energy.
\end{abstract}

Mathematics Subject Classification (2010). 74B05, 81P10.

Keywords. Elastic solid, rotational waves, transverse waves, Dirac equation.

\section{Introduction}

The ideal elastic solid has been an important model in the history of physics. It is a good approximation for many processes in condensed matter. It was also the basis of early theories of light because of its ability to support transverse waves [14]. Aether models fell into disuse at the end of the $19^{\text {th }}$ century when experiments failed to detect variations in light speed relative to the direction of the earth's motion through space [8]. However, de Broglie's wave hypothesis for matter [2] predicts a null result for such aether-drift experiments whether or not the waves propagate through a material medium.

It was recently proposed that a lattice of elastic cells would yield fermions and gauge fields equivalent to the Standard Model of particle physics [12]. This mechanical model is similar to the one James Maxwell used to develop the equations of electromagnetism [14]. While a homogeneous elastic solid may be regarded as a continuum limit of such a lattice, it is fundamentally simpler in structure.

It is current practice to describe physical events in terms of discrete elementary particles rather than continuous waves. However, the Dirac and 
Klein-Gordon equations which are used to compute the correlations between particle events also determine the wave-like evolution of continuously distributed physical quantities such as momentum, energy, and angular momentum densities $[13,5]$. Furthermore, the first-order Dirac equation may be regarded as a representation of a second-order wave equation with the bispinor wave function encoding the first derivatives $[7,9,10,11,3]$. Halfinteger spin is attributable to the fact that waves propagating in opposite directions are independent states separated by 180-degree rotation. Thus it is quite possible that a single wave equation may determine the evolution of dynamical quantities everywhere in space.

An ideal homogeneous, isotropic, elastic solid (with no dissipation and constant elasticity) is the simplest possible model for wave-like processes in three spatial dimensions. Hence the physics of an ideal elastic solid should be the foundation for any analysis of Lorentz-covariant processes in threedimensional space. Yet this simple model has never been properly understood.

Prior analyses of the elastic solid rely on approximations of infinitesimally small rotations or small rates of variation of rotations [6]. Analysis of first-order stress and strain yields an elastic wave equation for displacement $\mathbf{a}(\mathbf{r}, t)$ :

$$
\rho \partial_{t}^{2} \mathbf{a}=(2 \mu+\lambda) \nabla[\nabla \cdot \mathbf{a}]-\mu \nabla \times[\nabla \times \mathbf{a}] .
$$

This equation has two obvious flaws. First, since the displacement field is defined with respect to positions in space rather than particular elements of the solid, the value of the field is transported with the solid as it moves. Inclusion of convection by velocity $\mathbf{u}$ and rotation by vorticity $\mathbf{w}$ yields:

$$
\rho \partial_{t}^{2} \mathbf{a}+\rho \mathbf{u} \cdot \nabla \partial_{t} \mathbf{a}-\rho \mathbf{w} \times \partial_{t} \mathbf{a}=(2 \mu+\lambda) \nabla[\nabla \cdot \mathbf{a}]-\mu \nabla \times[\nabla \times \mathbf{a}] .
$$

The second flaw is more problematic. A rigid rotation by angle $\Theta$ yields a non-zero divergence of the displacement field $(\nabla \cdot \mathbf{a}=2(\cos \Theta-1))$. If $\Theta$ varies with position either radially or along the rotation axis, then the motion preserves volume and the elastic response should be proportional to the shear modulus $\mu$. Instead, the term $(2 \mu+\lambda) \nabla[\nabla \cdot \mathbf{a}]$ fails to distinguish between compressional and rotational origins of divergence of the displacement field. This is why the equation is only valid for infinitesimally small gradients of rotation.

The goal of this paper is to formulate an exact description of rotational (or transverse) waves in an ideal elastic solid. We will accomplish this task by describing incompressible motion in terms of rotations rather than displacements.

\section{Basic Assumptions}

We make the following basic assumptions:

1. The elastic solid is characterized by an inertial density $\rho$ and coefficient of elasticity $\mu$, with characteristic wave speed $c=\sqrt{\mu / \rho}$.

2. There is a linear response to variations of orientation angle $\Theta$ relative 
to equilibrium. This means that an initial static perturbation (with velocity $\mathbf{u}(\mathbf{r})=0$ ) would yield the response:

$$
\partial_{t}^{2} \Theta=c^{2} \nabla^{2} \Theta
$$

3. The velocity field $\mathbf{u}$ has no divergence $(\nabla \cdot \mathbf{u}=0)$. Therefore the velocity may be written as the curl of a vector field:

$$
\mathbf{u}=\frac{1}{2 \rho}[\nabla \times \overline{\mathbf{J}}] .
$$

The vector field $\overline{\mathbf{J}}$ is called the conjugate angular momentum density. It differs from the usual definition of angular momentum density $\mathbf{J}=\mathbf{r} \times \rho \mathbf{u}$ in that it is independent of the choice of origin and can have arbitrary direction. If $\mathbf{u}$ falls to zero sufficiently rapidly toward infinity, then integration by parts yields the alternative expressions for kinetic energy:

$$
K=\frac{1}{2} \int d^{3} r \rho u^{2}=\frac{1}{2} \int d^{3} r \mathbf{w} \cdot \overline{\mathbf{J}}
$$

where $\mathbf{w}=\nabla \times \mathbf{u} / 2$ is the angular velocity, or vorticity. Hence $\overline{\mathbf{J}}$ is the variable conjugate to angular velocity for a Lagrangian which depends on $\mathbf{u}$ only through the (positive) kinetic energy.

4. We assume that there are no velocity-dependent forces (such as frictional damping). Hence velocity only enters the equations of evolution through the convection and rotation of fields. We neglect any effect of the symmetric tensor $\left(\partial_{i} u_{j}+\partial_{j} u_{i}\right)$ on the orientation angle or angular momentum direction.

Additional assumptions will be introduced in order to simplify the mathematics, and these may limit the generality of the results.

\section{Equation of Evolution}

Starting from (3), we define an angular potential $\mathbf{Q}$ such that: $\nabla^{2} \mathbf{Q}=-4 \rho \mathbf{\Theta}$. The static condition for $\mathbf{Q}$ is:

$$
\nabla^{2}\left\{\partial_{t}^{2} \mathbf{Q}-c^{2} \nabla^{2} \mathbf{Q}\right\}=0 \quad(\text { if } \mathbf{u}(\mathbf{r})=0 \text { everywhere }) .
$$

Define the spin angular momentum as:

$$
\mathbf{S} \equiv \partial_{t} \mathbf{Q} .
$$

When motion is present, it contributes to the time derivative $\partial_{t} \mathbf{S}$ only through convection $(-\mathbf{u} \cdot \nabla \mathbf{S})$ and rotation $(\mathbf{w} \times \mathbf{S})$ :

$$
\nabla^{2}\left\{\partial_{t}^{2} \mathbf{Q}-c^{2} \nabla^{2} \mathbf{Q}+\mathbf{u} \cdot \nabla \mathbf{S}-\mathbf{w} \times \mathbf{S}\right\}=0 .
$$

From here on, we will consider only wave-like solutions satisfying:

$$
\partial_{t}^{2} \mathbf{Q}-c^{2} \nabla^{2} \mathbf{Q}+\mathbf{u} \cdot \nabla \mathbf{S}-\mathbf{w} \times \mathbf{S}=0 .
$$

For oscillatory solutions to this equation, the first two terms are always in phase $\left(\partial_{t}^{2} \mathbf{Q}-c^{2} \nabla^{2} \mathbf{Q}\right)$, whereas the velocity-dependent terms may have different phase. However, if the velocity-dependent terms do not add to zero 
then they must have the same temporal phase as the linear terms:

$$
\mathbf{u} \cdot \nabla \mathbf{S}-\mathbf{w} \times \mathbf{S}=\Omega^{2}(\mathbf{r}) \mathbf{Q}
$$

where $\Omega^{2}(\mathbf{r})$ is some function of position (more generally, $\Omega^{2}(\mathbf{r})$ could have different values for each component of $\mathbf{Q})$. Substitution yields:

$$
\partial_{t}^{2} \mathbf{Q}-c^{2} \nabla^{2} \mathbf{Q}+\Omega^{2}(\mathbf{r}) \mathbf{Q}=0 .
$$

If $\Omega^{2}(\mathbf{r})$ is constant and positive, then this is the Klein-Gordon equation.

Now our only remaining task is to solve for the velocity in terms of other wave variables. To do this we note that the above equation (9) can be written in terms of a four-component complex Dirac bispinor $(\psi)$ using the following identifications:

$$
\begin{aligned}
S_{j} \equiv \partial_{t} Q_{j} & \equiv \frac{1}{2}\left[\psi^{\dagger} \sigma_{j} \psi\right] \\
c[\nabla \cdot \mathbf{Q}] & \equiv-\frac{1}{2}\left[\psi^{\dagger} \gamma^{5} \psi\right] \\
c^{2}\{\nabla \times \nabla \times \mathbf{Q}\}_{j} & \equiv-\frac{\mathrm{i}}{2} c \epsilon_{i j k}\left\{\left[\partial_{i} \psi^{\dagger}\right] \gamma^{5} \sigma_{k} \psi-\psi^{\dagger} \gamma^{5} \sigma_{k} \partial_{i} \psi\right\} .
\end{aligned}
$$

This identification between bispinors and classical variables was made previously [4], where it was also assumed that the velocity could be derived from the spin. In this paper we will derive an expression for velocity from the conjugate momentum.

The matrices $\sigma_{j}$ are the Dirac spin matrices:

$$
\begin{aligned}
\sigma_{1} & =\left(\begin{array}{cccc}
0 & 1 & 0 & 0 \\
1 & 0 & 0 & 0 \\
0 & 0 & 0 & 1 \\
0 & 0 & 1 & 0
\end{array}\right), \sigma_{2}=\left(\begin{array}{cccc}
0 & -\mathrm{i} & 0 & 0 \\
\mathrm{i} & 0 & 0 & 0 \\
0 & 0 & 0 & -\mathrm{i} \\
0 & 0 & \mathrm{i} & 0
\end{array}\right), \\
\sigma_{3} & =\left(\begin{array}{cccc}
1 & 0 & 0 & 0 \\
0 & -1 & 0 & 0 \\
0 & 0 & 1 & 0 \\
0 & 0 & 0 & -1
\end{array}\right) .
\end{aligned}
$$

The matrices $c \gamma^{5} \sigma_{j}$ are the Dirac velocity matrices. One representation for $\gamma^{5}$ is:

$$
\gamma^{5}=\left(\begin{array}{cccc}
0 & 0 & 1 & 0 \\
0 & 0 & 0 & 1 \\
1 & 0 & 0 & 0 \\
0 & 1 & 0 & 0
\end{array}\right)
$$

The above identifications provide seven constraints on the eight free parameters of the Dirac bispinor. In terms of bispinors, the rotational wave equation (9) is:

$$
\begin{aligned}
\frac{\partial}{\partial t}\left[\psi^{\dagger} \sigma_{j} \psi\right] & +c \partial_{j}\left[\psi^{\dagger} \gamma^{5} \psi\right]-\mathrm{i} c \epsilon_{i j k}\left\{\partial_{i} \psi^{\dagger} \gamma^{5} \sigma_{k} \psi-\psi^{\dagger} \gamma^{5} \sigma_{k} \partial_{i} \psi\right\} \\
& +\mathbf{u} \cdot \nabla\left[\psi^{\dagger} \sigma_{j} \psi\right]-\epsilon_{k i j} w_{k}\left[\psi^{\dagger} \sigma_{i} \psi\right]=0
\end{aligned}
$$


Expanding the derivatives yields:

$$
\psi^{\dagger} \sigma_{j}\left[\partial_{t} \psi+c \gamma^{5} \boldsymbol{\sigma} \cdot \nabla \psi+\mathbf{u} \cdot \nabla \psi+\mathbf{w} \cdot \frac{\mathrm{i} \boldsymbol{\sigma}}{2} \psi\right]+\text { c.c. }=0
$$

where (c.c.) represents the complex conjugate. The Hermitian conjugate wave function may be regarded as an independent variable (the independent real and imaginary parts of the wave function are linear combinations of elements of $\psi$ and $\psi^{\dagger}$ ). Validity for arbitrary $\psi^{\dagger}$ requires the terms in brackets to sum to zero. This yields the Dirac equation:

$$
\partial_{t} \psi+c \gamma^{5} \boldsymbol{\sigma} \cdot \nabla \psi+\mathbf{u} \cdot \nabla \psi+\mathrm{iw} \cdot \frac{\boldsymbol{\sigma}}{2} \psi+\mathrm{i} \chi \psi=0
$$

where $\chi$ may be any operator with the property:

$$
R e\left(\psi^{\dagger} \sigma_{j} \mathrm{i} \chi \psi\right)=0 .
$$

Since $\chi$ has no effect on the original equation for $\mathbf{Q}$, we assume it to be zero.

Now we construct a Lagrange density $\mathscr{L}$. Lagrange's equations of motion for a field variable $\psi$ are:

$$
\partial_{t} \frac{\partial \mathscr{L}}{\partial\left[\partial_{t} \psi\right]}+\sum_{j} \partial_{j} \frac{\partial \mathscr{L}}{\partial\left[\partial_{j} \psi\right]}-\frac{\partial \mathscr{L}}{\partial \psi}=0
$$

A similar equation holds with $\psi^{\dagger}$ replacing $\psi$. A possible Lagrange density for rotational waves is therefore:

$$
\mathscr{L}=-\mathrm{i} \psi^{\dagger} \partial_{t} \psi+\psi^{\dagger}\left[-\mathrm{i} c \gamma^{5} \boldsymbol{\sigma} \cdot \nabla\right] \psi+\psi^{\dagger}\left[-\mathrm{i} \mathbf{u} \cdot \nabla+\mathbf{w} \cdot \frac{\boldsymbol{\sigma}}{2}\right] \psi .
$$

Using the Hermitian conjugate of (19) yields simply $\partial \mathscr{L} / \partial \psi^{\dagger}=0$. Since the complex conjuate of the equation of evolution is also valid, we can require the Lagrange density to be real:

$$
\mathscr{L}=\operatorname{Re}\left\{-\mathrm{i} \psi^{\dagger} \partial_{t} \psi+\psi^{\dagger}\left[-\mathrm{i} c \gamma^{5} \boldsymbol{\sigma} \cdot \nabla\right] \psi+\psi^{\dagger}\left[-\mathrm{i} \mathbf{u} \cdot \nabla+\mathbf{w} \cdot \frac{\boldsymbol{\sigma}}{2}\right] \psi\right\} .
$$

The conjugate momentum to the field $\psi$ is $p_{\psi}$ :

$$
p_{\psi}=\frac{\partial \mathscr{L}}{\partial\left[\partial_{t} \psi\right]}=-\mathrm{i} \psi^{\dagger} .
$$

We assume that we can neglect boundary terms in the integration by parts of:

$$
\int d^{3} r \mathbf{w} \cdot \mathbf{S}=\frac{1}{2} \int d^{3} r[\nabla \times \mathbf{u}] \cdot \mathbf{S}=\frac{1}{2} \int d^{3} r \mathbf{u} \cdot[\nabla \times \mathbf{S}] .
$$

The conjugate momentum for $\mathbf{r}$ is:

$$
\mathbf{p}_{\mathbf{r}}=\frac{\partial \mathscr{L}}{\partial \mathbf{u}}=\operatorname{Re}\left(-\psi^{\dagger} \mathrm{i} \nabla \psi\right)+\frac{1}{2} \nabla \times \psi^{\dagger} \frac{\boldsymbol{\sigma}}{2} \psi=\rho \mathbf{u} .
$$

This conjugate momentum was derived under the assumption that $\mathbf{u}$ is an independent variable. Identification of $\mathbf{p}_{r}$ with $\rho \mathbf{u}$ is justified by the lack of external forces, implying that $\rho \mathbf{u}$ enters the Lagrangian only through the kinetic energy. 
Making $\mathbf{u}$ a function of $\psi$ introduces a factor of $1 / 2$ :

$$
\begin{aligned}
\mathscr{L} & =\operatorname{Re}\left\{-\mathrm{i} \psi^{\dagger} \partial_{t} \psi+\psi^{\dagger}\left[-\mathrm{i} c \gamma^{5} \boldsymbol{\sigma} \cdot \nabla\right] \psi\right\} \\
& +\frac{1}{2 \rho}\left[\operatorname{Re}\left(-\psi^{\dagger} \mathrm{i} \nabla \psi\right)+\frac{1}{2} \nabla \times \psi^{\dagger} \frac{\boldsymbol{\sigma}}{2} \psi\right]^{2} .
\end{aligned}
$$

Variation of this Lagrange density determines the evolution of rotational waves:

$$
\partial_{t} \psi+c \gamma^{5} \boldsymbol{\sigma} \cdot \nabla \psi+\mathbf{u} \cdot \nabla \psi+\mathrm{iw} \cdot \frac{\boldsymbol{\sigma}}{2} \psi=0
$$

where velocity $\mathbf{u}$ and vorticity $\mathbf{w}=\nabla \times \mathbf{u} / 2$ are functions of $\psi$ as determined from (24).

\section{Dynamical Variables}

\subsection{Angular Momentum}

Recall that the velocity is the curl of an origin-independent angular momentum:

$$
\mathbf{u}=\frac{1}{2 \rho} \nabla \times \overline{\mathbf{J}}
$$

We can separate the conjugate angular momentum $(\overline{\mathbf{J}})$ into orbital $(\overline{\mathbf{L}})$ and spin $(\mathbf{S})$ components by identifying the orbital $\left(\mathbf{u}_{\mathbf{L}}\right)$ and spin $\left(\mathbf{u}_{\mathbf{S}}\right)$ contributions to velocity:

$$
\begin{aligned}
& \mathbf{u}_{\mathbf{L}}=\frac{1}{\rho} \operatorname{Re}\left(-\psi^{\dagger} \mathrm{i} \nabla \psi\right)=\frac{1}{2 \rho} \nabla \times \overline{\mathbf{L}} \\
& \mathbf{u}_{\mathbf{S}}=\frac{1}{2 \rho} \nabla \times \psi^{\dagger} \frac{\boldsymbol{\sigma}}{2} \psi=\frac{1}{2 \rho} \nabla \times \mathbf{S} .
\end{aligned}
$$

The incompressibility condition $\nabla \cdot \mathbf{u}=0$ places an additional restriction on the wave function:

$$
\nabla \cdot \rho \mathbf{u}_{\mathbf{L}}=\frac{1}{2} \nabla \cdot\left[-\psi^{\dagger} \mathrm{i} \nabla \psi+\mathrm{i}[\nabla \psi] \psi\right]=\frac{\mathrm{i}}{2}\left\{-\psi^{\dagger} \nabla^{2} \psi+\left[\nabla^{2} \psi^{\dagger}\right] \psi\right\}=0 .
$$

The usual definition of angular momentum $\mathbf{J}=\mathbf{L}+\mathbf{S}$ depends on the choice of origin. If we define a rotational velocity as $\mathbf{u}_{\mathrm{R}}=\mathbf{w} \times \mathbf{r}$ and note that the vorticity is the instantaneous angular velocity $(\mathbf{w}=d \mathbf{\Theta} / d t)$, then from (19) the conjugate angular momentum would have the same form as found in relativistic quantum mechanics:

$$
\mathbf{p}_{\Theta}=\frac{\partial \mathscr{L}}{\partial\left[\partial_{t} \boldsymbol{\Theta}\right]}=\psi^{\dagger}\left\{-\mathbf{r} \times \mathrm{i} \nabla+\frac{\boldsymbol{\sigma}}{2}\right\} \psi=\mathbf{L}+\mathbf{S} .
$$

\subsection{Energy and momentum}

In the Lagrange density defined in (25), the velocity-dependent term is clearly the kinetic energy density. This observation suggests that the Lagrangian has the form:

$$
\mathscr{L}=\operatorname{Re}\left\{-\mathrm{i} \psi^{\dagger} \partial_{t} \psi+\psi^{\dagger}\left[-\mathrm{i} c \gamma^{5} \boldsymbol{\sigma} \cdot \nabla\right] \psi+\frac{1}{2} \rho u^{2}\right\}=-\mathscr{E}+U+K
$$


where $\mathscr{E} \equiv \operatorname{Re}\left(\mathrm{i} \psi^{\dagger} \partial_{t} \psi\right)$ is the total energy, $U \equiv \operatorname{Re}\left(-\psi^{\dagger}\left[\mathrm{i} c \gamma^{5} \boldsymbol{\sigma} \cdot \nabla\right] \psi\right)$ is potential energy, and $K$ is kinetic energy density. We will simply adopt these definitions without further justification.

The Hamiltonian is the negative of the energy:

$$
\begin{aligned}
\mathscr{H}=p_{\psi} \partial_{t} \psi-\mathscr{L} & =\operatorname{Re}\left\{\psi^{\dagger}\left[\mathrm{i} c \gamma^{5} \boldsymbol{\sigma} \cdot \nabla+\frac{1}{2}\left(\mathrm{iu} \cdot \nabla-\mathbf{w} \cdot \frac{\boldsymbol{\sigma}}{2}\right)\right] \psi\right\} \\
& =-\left\{U+\frac{1}{2} \rho u^{2}\right\}
\end{aligned}
$$

Hamilton's equation for the wave function is:

$$
\partial_{t} \psi=\frac{\partial \mathscr{H}}{\partial p_{\psi}}=\frac{\partial \mathscr{H}}{\partial\left[-\mathrm{i} \psi^{\dagger}\right]}=\left\{-c \gamma^{5} \boldsymbol{\sigma} \cdot \nabla-\mathbf{u} \cdot \nabla-\mathrm{iw} \cdot \frac{\boldsymbol{\sigma}}{2}\right\} \psi .
$$

We can also define a Hamiltonian operator with $\partial_{t} \psi=\mathrm{i} H \psi$ (note opposite sign convention from quantum mechanics):

$$
H=\mathrm{i} c \gamma^{5} \boldsymbol{\sigma} \cdot \nabla+\mathrm{i} \mathbf{u} \cdot \nabla-\mathbf{w} \cdot \frac{\boldsymbol{\sigma}}{2} .
$$

The Hamiltonian is a special case $\left(T_{0}^{0}\right)$ of the energy-momentum tensor:

$$
T_{\nu}^{\mu}=\frac{\partial \mathscr{L}}{\partial\left[\partial_{\mu} \psi\right]} \partial_{\nu} \psi-\mathscr{L} \delta_{\nu}^{\mu} .
$$

The dynamical momentum density $\mathbf{P}$ is derived from the orbital part of the angular momentum:

$$
T_{i}^{0}=P_{i}=-\mathrm{i} \psi^{\dagger} \partial_{i} \psi
$$

This is identical to the dynamical momentum of relativistic quantum mechanics. Unlike the conjugate momentum, it does not include the rotational motion associated with the spin angular momentum.

The sign of the Hamiltonian (and Lagrangian) is simply a convention. We chose signs to make the conjugate momentum parallel (rather than antiparallel) to velocity, thus requiring the Hamiltonian to be the negative of the energy. In analogy with plane waves, the function $\cos (\omega t-k x)$ with $\omega>0$ is equivalent to $\cos (\omega t+k x)$ with $\omega<0$. We could change the sign of the Lagrangian and Hamiltonian and still preserve the sign of the momentum by using covariant derivatives $\left(\partial_{\mu}=\left(\partial_{t},-\partial_{i}\right)\right)$. However, such a relativistic construction is unnecessary and perhaps misleading since we are in fact dealing with Galilean space-time (the laws of relativity apply to the space of measurements, not to the absolute space-time). The energy-momentum tensor components $T_{\mu}^{0}=\left(-\mathscr{E}, P_{i}\right)$ may still be regarded as a covariant vector whose magnitude is the scalar $\mathscr{E}^{2}-P^{2}$. The equation of evolution is of course unaffected by the choice of sign of the Lagrangian.

\section{Discussion}

The description of rotational waves derived here provides a simple classical interpretation of Dirac bispinors and quantum mechanical operators. Due to the nonlinear nature of the equation of evolution, we expect that an elastic 
solid has a discrete spectrum of fermionic soliton solutions. If each soliton is assigned its own independent wave function, then cancellation of interference between these independent 'particles' would yield the Pauli exclusion principle and require the introduction of interaction potentials [4]. Characterization of these interaction potentials is beyond the scope of this paper.

Correlations between rotational soliton waves would be computed in the same manner as in relativistic quantum mechanics [4]. The reason is that the bispinor wave functions, and not the measurement values, satisfy a first order wave equation which may be used to directly compute variations in space and time. Therefore Bell's Theorem [1] is not applicable.

The quantum mechanical Dirac equation is also equivalent to a deterministic equation for the evolution of spin angular momentum density. Comparison of the classical and quantum equations for spin density may yield new understanding of the physical properties of elementary particles.

\section{Conclusions}

A nonlinear Dirac equation describes evolution of rotational waves in an ideal isotropic elastic solid. The equation is derived by assuming a linear response to variations in orientation and incorporating the influence of motion through convection and rotation. The Hamiltonian is represented as a sum of potential and kinetic energy. This result provides a simple classical interpretation of Dirac bispinors and the associated dynamical operators of relativistic quantum mechanics.

\section{Acknowledgment}

The author is grateful to Damon Merari for his interest and encouragement.

\section{References}

[1] J. S. Bell, On the Einstein Podolsky Rosen Paradox. Phys. 1 (1964), 195-200.

[2] L. de Broglie, Recherches sur la Théorie des Quanta. PhD Thesis, University of Sorbonne, Paris, 1924.

[3] R. A. Close, Torsion waves in three dimensions: quantum mechanics with a twist. Found. Phys. Lett. 15 (2002), 71-83.

[4] R. A. Close, A Classical Dirac Equation. In: Ether Space-time \& Cosmology, Vol 3. (Physical vacuum, relativity, and quantum physics) eds. M.C. Duffy and J. Levy, Apeiron, Montreal 2009, 49-73.

[5] D. Hestenes, Local observables in the Dirac theory. J. Math. Phys. 14 (7) (1973), 893-905.

[6] H. Kleinert, Gauge Fields in Condensed Matter. vol II. World Scientific, Singapore, 1989, 1240-64.

[7] S. Matsutani and H. Tsuru, Physical relation between quantum mechanics and solitons on a thin elastic rod. Phys. Rev. A 46 (1992), 1144-7.

[8] A. A. Michelson and E. W. Morley, On the relative motion of the earth and the luminiferous ether. Am. J. Sci. (3rd series) 34 (1887), 333-45. 
[9] P. Rowlands, The physical consequences of a new version of the Dirac equation. In: Causality and Locality in Modern Physics and Astronomy: Open Questions and Possible Solutions (Fundamental Theories of Physics, vol 97) eds G. Hunter, S. Jeffers, and J-P. Vigier, Kluwer Academic Publishers, Dordrecht, 1998, 397-402.

[10] P. Rowlands, J. P. Cullerne, The connection between the Han-Nambu quark theory, the Dirac equation and fundamental symmetries. Nuclear Phys. A 684 (2001), 713-715.

[11] P. Rowlands, Removing redundancy in relativistic quantum mechanics. Preprint: arXiv:physics/0507188 (2005).

[12] I. Schmelzer, A condensed matter interpretation of SM fermions and gauge fields. Found. Phys. 39 (1) (2009), 73-107.

[13] Y. Takabayashi, Relativistic hydrodynamics of the Dirac matter. Suppl. Prog. Theor. Phys. 4 (1) (1957), 1-80.

[14] E. Whittaker, A History of the Theories of Aether and Electricity. Vol 1. Thomas Nelson and Sons Ltd., Edinburgh, 1951, 128-69.

R. A. Close

4110 SE Hawthorne Blvd. \#232

Portland, OR 97214

USA

e-mail: robert.close@classicalmatter.org

Received: July 16, 2009.

Accepted: October 8, 2009.

Open Access This article is distributed under the terms of the Creative Commons Attribution Noncommercial License which permits any noncommercial use, distribution, and reproduction in any medium, provided the original author(s) and source are credited. 